\title{
SPORT BEI ZWILLINGEN
}

\author{
von \\ Hans Grebe
}

In der noch jungen Sportgeschichte unseres Jahrhunderts finden sich bei besonders erfolgreichen Vertretern einer Sportart nicht selten gleiche Namen und unter diesen vielfach nahe Blutsverwandte - einige Male auch Zwillinge. Man möchte hieraus eine Unterlage für die Annahme sehen, dass die sportliche Leistungsfähigkeit und insbesondere die Befähigung zu sportlichen Spitzenleistungen zumindest von der Erbveranlagung des einzelnen abhängig ist.

Anderseits wird man nach den besonderen Erfolgen der durch höchstes Training geschulten Sportler der grossen Sportnationen der sportlichen Schulung als Umweltwirkung eine ausschlaggebende Bedeutung für die Steigerung der sportlichen Leistungsfähigkeit zuerkennen wollen. Dies gilt in erster Linie für die Entwicklung der russischen Sportler, die in den letzten 5 Jahren kometengleich aufgestiegen sind und in vielen sportlichen Disziplinen der absoluten Leistungsgrenze nahe gekommen sind. Man darf aber gerade bei den Leistungen der russischen Sportler nicht vergessen, dass in Russland der Staat und seine Körperschaften seit einigen Jahren eine systematische Suche nach sportlichen Talenten auf breitester Basis durchführen und dass erst aus einer grossen Zahl besonders ausgesuchter und geförderter "Talente" die Spitzensportler und ihre zunächst überraschenden phänomenalen Leistungen unter härtesten Auslesebedingungen resultieren. Es müssen also auch bei den Sportlern aus Russland und anderen Ländern mit staatlicher oder besonders grosser privater Unterstützung des Sportes und scharf auslesenden Trainingsbedingungen zunächst Anlagen zu sportlicher Leistungsfähigkeit vorhanden sein, die in der Erbmasse, also im Genotypus, begründet sind.

Erbe und Umwelt mögen aber auch in berühmten Sportfamilien wie bei den norwegischen Brüdern Ruud (Skispringen), den französischen Geschwistern Couttet (-Schmitt), den Tiroler Geschwistern Lantschner und den Freiburger Geschwistern Cranz (alpine Skiwettbewerbe), den US-amerikanischen Brüdern Jenkins und den kanadischen Geschwistern Kennedy (Eiskunstlauf), den deutschen Brüdern Walter (Fussball) oder auch den besten belgischen Tennisspielern aller Zeiten Jean und Philipp Washer (Vater und Sohn), aber auch bei Berufssportlern wie den holländischen "Sechstagekönigen " Piet und Jan van Kempen oder den kanadischen Eishockytrainern Lorne und Frank Trottier (jeweils Brüder) und vielen anderen zusammenwirken. Wird doch in Sportlerfamilien unabhängig von der erblichen Veranlagung zu sportlicher Leistungsfähigkeit auch die Erziehung und der Drang, einem Vorbild nachzueifern, leistungsbegünstigend sein. 
Aus einer solchen Kombination zwischen sportlicher Veranlagung und Erziehung wird eine Meldung besonders verständlich, die erst kürzlich durch Presse und Funk ging: Bei den Weltmeisterschaften im modernen Fünfkampf 1954 siegte der schwedische Kadett Björn Thofeldt, dessen Vater Sven Thofeldt 1928 Olympiasieger in der gleichen Vielseitigkeitsprüfung war.

Um eine tatsächlich verwertbare Grundlage für das Zusammenwirken von Erbanlage und Umwelt für die aktive sportliche Betätigung wie für die Erzielung besonderer sportlicher Leistungen zu erhalten, habe ich im im Rahmen eines von mir auf dem Deutschen Sportärztekongress 1954 erbetenen Referates im letzten Jahr versucht, eine möglichst auslesefreie Zwillingsuntersuchung durchzuführen, über deren vorläufige Ergebnisse im folgenden berichtet werden soll:

Vorwegnehmen möchte ich noch, dass mir eine entsprechende Untersuchung von irgendwelcher anderen Seite bisher nicht bekannt geworden ist. Meine Befunde möchte ich deshalb zur Diskussion stellen. Mögen sie entsprechende und ausgedehntere Untersuchungen veranlassen!

Zur Erzielung einer möglichst auslesefreien Zwillingsserie wandte ich mich über die "Hessischen Sportmitteilungen" an sämtliche Sportvereine Hessens. Aus den eingegangenen Meldungen von "sporttreibenden Zwillingen» schied ich die Pärchen-Zwillinge aus, da die Geschlechtsverschiedenheit allein schon als ausschlaggebende Ursache für diskordantes sportliches Verhalten gelten könnte. Für meine Erhebungen verwertbar und von mir untersucht werden konnten bisher 34 gleichgeschlechtige Paare, deren Befunde sich aus der folgenden Tabelle ergeben:

Tabelle I

\begin{tabular}{c|c|c|c||c|c|c|}
\cline { 2 - 7 } & \multicolumn{3}{|c||}{ EZ } & \multicolumn{3}{c|}{ ZZ } \\
\cline { 2 - 7 } & $\mathrm{K}$ & $\mathrm{KD}$ & $\mathrm{D}$ & $\mathrm{K}$ & $\mathrm{KD}$ & $\mathrm{D}$ \\
\hline $0^{\star}$ & 12 & 1 & 0 & 4 & 2 & 1 \\
\hline 9 & 8 & 0 & 0 & 3 & 2 & 1 \\
\hline \hline Summe & 20 & 1 & 0 & 7 & 4 & 2 \\
\hline
\end{tabular}

Aus dieser Tabelle geht hervor, dass von 13 männlichen EZ-Paaren nur ein Paar (EZ 12) bei ähnlich starkem sportlichem Interesse wesentliche Verschiedenheiten in der Art der sportlichen Betätigung wie der sportlichen Leistungsfähigkeit erkennen liess, während die 12 übrigen männlichen EZ-Paare völlige Konkordanz in der Einstellung zum Sport wie besonders in der Art der sportlichen Betätigung und der erzielten sportlichen Leistungshöhe zeigten. 
Von den 8 untersuchten weiblichen EZ waren alle völlig konkordant.

Gegenüber diesen auffällig hohen Uebereinstimmungen bei den EZ fanden sich bei den $\mathrm{ZZ}$ neben entsprechend starken Konkordanzen auch einige Paare mit mehr oder weniger grosser Diskordanz.

So zeigten von 7 männlichen ZZ-Paaren nur 4 völlig konkordantes Verhalten in der Ausübung einer Sportart wie besonders auch in dem bevorzugten Sportzweig und in der erzielten Leistungshöhe. Von den übrigen 3 Paaren zeigten 2 zwar in ähnlicher Weise das Verlangen nach sportlicher Aktivität, jedoch deutliche Verschiedenheiten in der wettkampfmässigen sportlichen Betätigung. Von den letzten ZZ-Brüdern war nur einer sportlich aktiv, während der andere wegen besonderer musischer Interessen weder sportlich hervorgetreten noch überhaupt am Sport interessiert war.

Bei den 6 untersuchten weiblichen ZZ-Paaren fanden sich ähnliche Verhältnisse. 3 in der Einstellung zum Sport wie in der Wettkampfart konkordanten ZZ-Schwestern stehen 2 Paare mit ähnlicher Bereitschaft zu sportlicher Aktivität, jedoch mit unterschiedlicher Erfüllung dieser Bereitschaft, und 1 Paar mit völlig divergierenden Interessen gegenüber. Dabei scheint es mir besonders bemerkenswert, dass unter den weiblichen ZZ-Paaren mit Diskordanz zweimal 1 Paarling eine deutsche Meisterschaft erringen konnte, während die Schwester entweder nur einer sportlichen Liebhaberei ohne Leistungsehrgeiz nachging oder dem Sport gegenüber völlig konträre Interessen zeigte.

Vor einer weiteren Diskussion der erhobenen Befunde seien diese selbst im Hinblick auf die sportliche Betätigung und die erzielten Höchstleistungen kurz angeführt:

\section{a. ERBGLEICHe ZWILlinge (EZ)}

\section{Männlich :}

EZ 11926 geboren. Bis zum 18. Lebensjahr im Elternhaus in Oberschlesien. Dann beide 1944-45 Soldat. I im Kriege am Fuss verwundet. Nach Gefangenschaft seit 1946 beide in Hessen. Während der Schul-und Lehrzeit trieben beide viel Leichtathletik. Vom 16.18. Lebensjahr und nach 1946 spielten beide Fussball, fast immer in der gleichen Mannschaft. Wegen seiner Kriegsverletzung wurde I (auf Bild rechts) Tormann, II ist Feldspieler (s. Abb. 1a).

EZ 21919 geboren. Seit 1925 Leichtathletik. Mit 14 Jahren 1. u. 2. Stadtmeister von Kassel im Leichtathletik-Dreikampf. In den folgenden Jahren wechselweise viele Leichtathletik-Siege mit fast gleicher Punktzahl. Seit dem 17. Lebensjahr beide aktive Fussballpieler: I Tormann, II Verteidiger und Mittelläufer. Beide Oberligastammspieler bis zum Kriegsausbruch 1939 und nach dem Krieg noch bis 1950 Vertragsspieler. I 1938 Ersatztormann in der damaligen deutschen Fussball-Ländermannschaft gegen Belgien, 1939 mehrfach repräsentativ für Hessen. Gleiche Berufsinteressen: I Bilanzbuchhalter, II Stadtinspektor. Die Eltern haben die sportliche Entwicklung der Brüder sehr gefördert (Abb. 1b).

EZ 31927 geboren. I Zimmermann, II Büroangestellter. In der Schule waren beide gute Schwimmer, Turner und Leichtathleten. Seit dem 16. Lebensjahr aktive Fussball- 


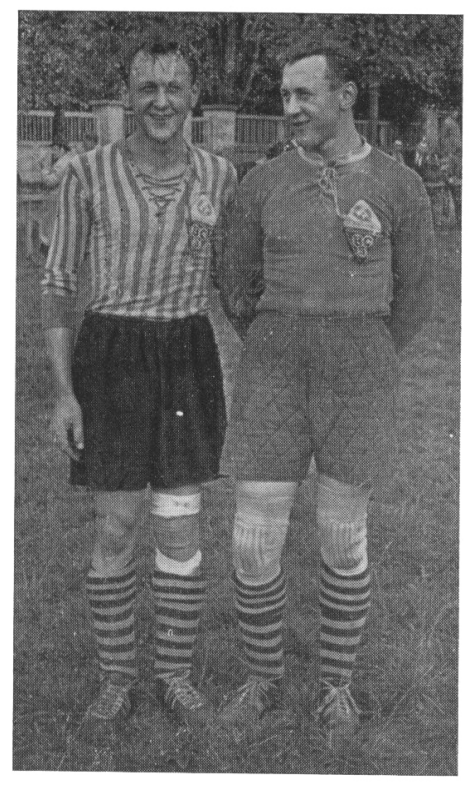

a) $\mathrm{EZ} 1$

spieler, z.Zt. beide in der gleichen Amateurligamannschaft, in der ausserdem noch ein älterer Bruder und 2 Vettern spielen! Beide Zwillingsbrüder waren stets Halb-oder Aussenstürmer. In der Familie zahlreiche Sportler, darunter die weiblichen Zwillinge EZ 17. Beide Zwillingsbrüder waren bis auf 3 Jahre Trennung durch Krieg und Gefangenschaft fast immer im Elternhaus.

EZ 41936 geboren. Beide sind kaufmännische Lehr. linge und spielen seit der Schulentlassung in der gleichen Jugend-Fussballmannschaft als Verteidiger. Beide leben im Elternhaus. Bei den Schwimmwettkämpfen der Stadt Wiesbaden belegten beide im letzten Schuljahr die beiden ersten Plätze. Der Vater war aktiver Fussballer.

EZ 51937 geboren. Wohnen im gleichen Stadtteil Wiesbadens wie EZ 4 und spielen auch in der gleichen Jugend-Fussballmannschaft wie EZ 4 als Halbstürmer mit Bevorzugung des linken Fusses. Der Vater spielte in Ligamannschaft Linksaussen.

EZ 61930 geboren. Seit Schulentlassung sind beide Angestellte in den Höchster Farbwerken. Seit 1949 konzentrierten sich bei beiden die bis dahin allgemeinen

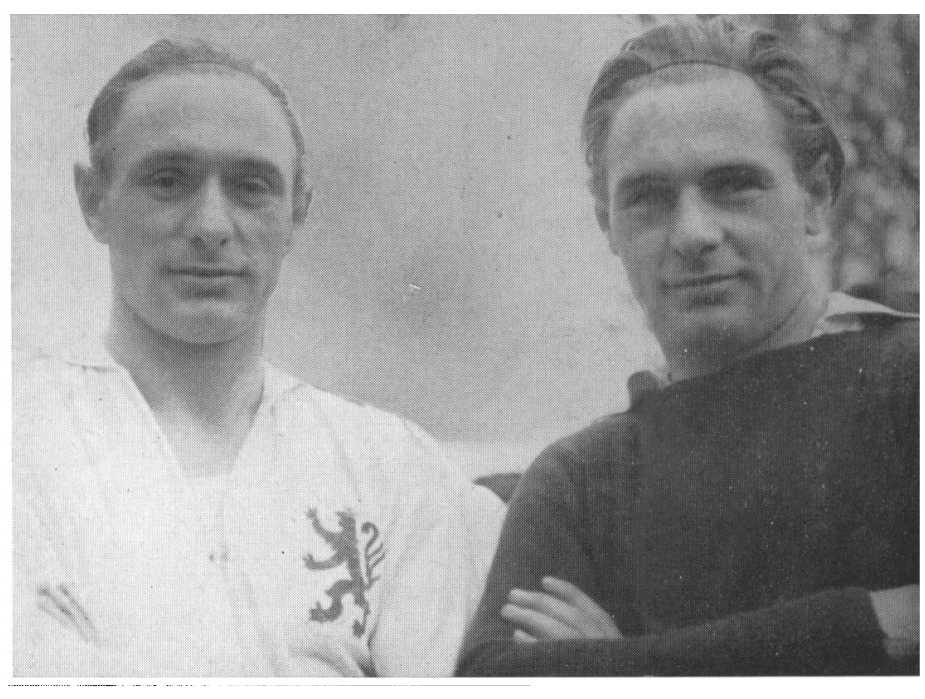

b) $\mathrm{EZ} 2$

Abb. $1 a-b$ - Erbgleiche Zwillinge, die in der gleichen Fusballmannschaft spielen. sportlichen Interessen auf Mittelstrecken - Laufwett. bewerbe. Beide trainieren seit dieser Zeit regelmässig gemeinsam und erzielten folgende Bestzeiten:

$400 \mathrm{~m}$ : I 52,0

II 52,5

$800 \mathrm{~m}:$ I $1.56,8$

II $1.57,1$

$1000 \mathrm{~m}: 12.32$

II 2.30

$1500 \mathrm{~m}: \mathrm{l} 4.01,2$

II $4.04,4$

Den grössten Erfolg hatten beide 1953-54 mit einem 3. u. 4. Platz bei den deutschen Hallenleichtathletikmeisterschaften im $800 \mathrm{~m}$ - Lauf (s. Abb. 2). 


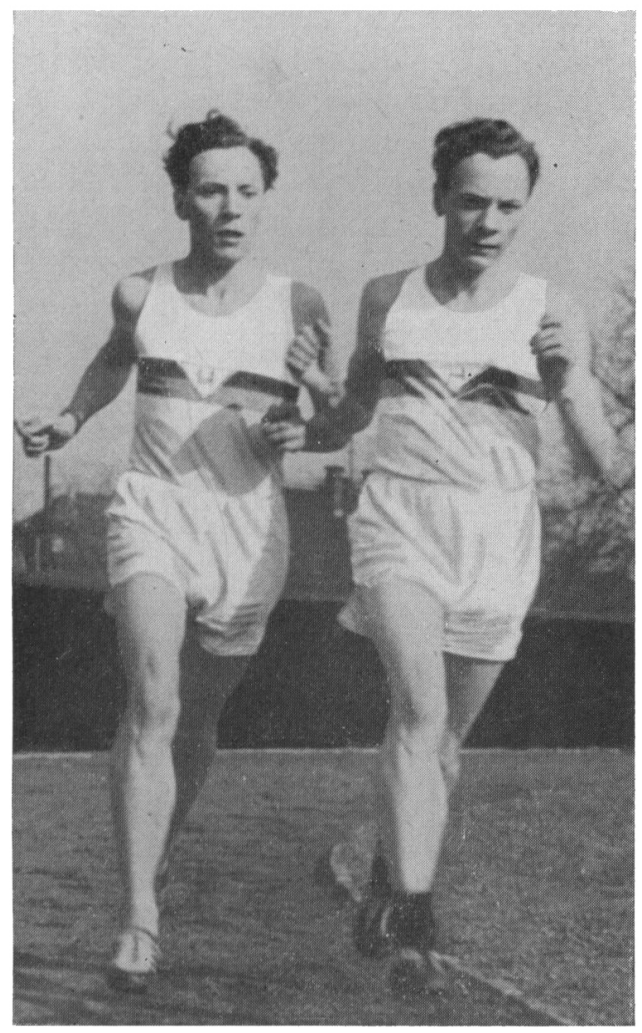

Abb. 2 - Erbgleiche Zwillinge mit gleicher Begabung für Mittelstreckenlauf (EZ 6)

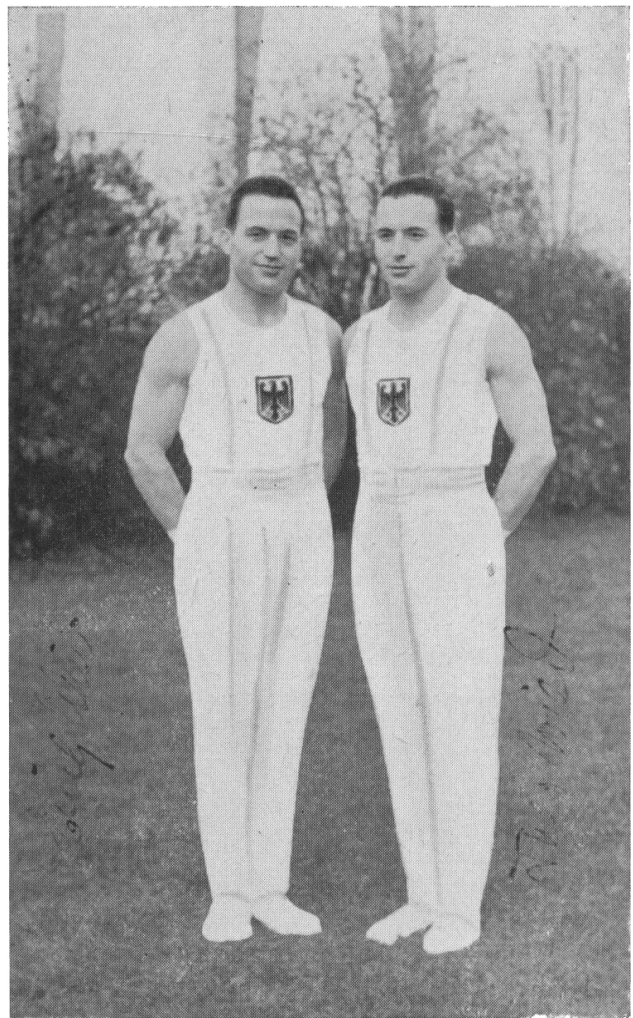

Abb. 3 - Erbgleiche Zwillinge, die seit 10 Jahren $z \mathrm{u}$ den besten deutschen Geräteturnern zählen und der Olympiariege angehören (EZ 8)

EZ 71934 geboren. Mit gleicher Leistungsstärke spielten beide während der Schulzeit Handball und Fussball. Seit dem 15. Lebensjahr sind beide aktive Leichtathleten. Daneben wiederholt Teilnahme an Schwimm- und Skikonkurrenzen. Bei den leichtathletischen Wettkämpfen zieht I heute den $400 \mathrm{~m}$-Lauf, II den Weitsprung vor. Beste Leistungen im Sommer 1954:
$100 \mathrm{~m}$ : I 11,9
Weitsprung: I 5,75
$400 \mathrm{~m}: \mathrm{I} 52,0$
Hochspr.: I 1,65
II 11,8
II 6,10
II 54,0
II 1,65

Beide trainieren ohne Anleitung. In der Familie keine besonderen sportlichen Leistungen.

EZ 81923 geboren. Beide sind von Beruf Maschinen-Ingenieure und Deutschlands bekannteste Sport-Zwillinge. 1941 wurden beide 2. deutsche Jugendmeister im Geräteturnen mit gleicher Punktzahl (!). Seit dieser Zeit gehören beide zu den besten Turnern Deutschlands, die 10 Jahre hintereinander jeweils unter den 5 Erstplazierten bei den 
Deutschen Turnmeisterschaften und ständige Angehörige der deutschen Nationalmannschaft und der Olympia-Mannschaft waren.

1943 war II deutscher Meister und I mit geringem Punktunterschied Zweiter. 1948 und 1949 belegten beide in umgekehrter Reihenfolge die ersten beiden Plätze und noch 1953 erreichten beide Platz 2 und 3. Beide Zwillingsbrüder haben sich damit 12 Jahre lang in völlig gleicher Weise an der Spitze der Deutschen Turner gehalten (s. Abb. 3).

In der väterlichen Familie waren mehrere hervorragende Schwerathleten, unter denen es der Bruder des Grossvaters 1911 bis zur Weltmeisterschaft gebracht hatte. Zwei Vettern der Zwillingsbrüder haben in der gleichen Fussballmannschaft gespielt, die nach 1948 zweimal deutscher Meister werden konnte.

EZ 91937 geboren. Seit dem 11. Lebensjahr errangen beide wiederholt bei Leichtathletikkämpfen erste Plätze. Beide sind kaufmännische Lehrlinge, spielen in der gleichen Handballmannschaft und gehören jetzt zu den besten Jugend-Geräteturnern!Nordhessens. Während 1953 bei Bezirkswettkämpfen I den ersten und II den zweiten Platz (mit 1/10 Punktdifferenz) belegen konnten, erreichte 1954 bei Gaugerätemeisterschaften II den ersten und I den 4. Platz. Die grössere Punktdifferenz führen beide auf einen Fehlgriff von I zurück. Die grosse Aehnlichkeit der beiden Brüder geht auch aus der wie bei EZ 8 sehr ähnlichen Haltung an den Geräten hervor. Der Vater war bis zum 35. Lebensjahr Wettkampfturner.

EZ 101926 geboren. Nach der Schulentlassung gemeinsame Lehrzeit als Modellbauer, anschliessend gemeinsam beim Arbeitsdienst und Militär. 1945-1946 in Gefangenschaft getrennt. Seitdem bis zur Verheiratung wieder zusammen. Beide aktive Boxer in der gleicher Staffel und Gewichtsklasse.

EZ 111934 geboren. Seit der Schulzeit Geräteturner und Leichtathleten. Besonders im Ballweitwurf hervorgetreten, erzielten nahezu gleiche Weiten. Beide spielen seit 2 Jahren in der gleichen Liga-Handballmannschaft als Stürmer. Im Sommer arbeiten beide in der elterlichen kleinen mittelhessischen Landwirtschaft, im Winter als Holzfäller im Walde.

EZ 121935 geboren. Bis zum Frühjahr 1945 völlig gleiche Umwelt im Elternhaus. Dann erlitt II durch Explosion einer bei Kriegsende gefundenen Handgranate eine schwere Verletzung der rechten Hand, von der nur noch der Daumen und der Kleinfinger und von den Fingern 2-4 etwa 2/3 der Mittelhandknochen erhalten geblieben sind. Seit dieser Zeit blieb II, besonders psychisch, jahrelang hinter I in der Entwicklung zurück. 1947 begannen beide Brüder, in der gleichen Schülermannschaft Fussball zu spielen. Seit 1948 reiten beide, deren Mutter früher Turnierreiterin war, jedoch ohne besondere Passion. Erst als die vermögenden Eltern 1950 einen eigenen Tennisplatz bauten, begann II den damals grossen Vorsprung seines Bruders aufzuholen. Obwohl er damals als Rechtshänder auf die gesunde linke Hand umschulen musste, ist es ihm jetzt durch fleissiges Training gelungen, die Leistungsfähigkeit seines gesunden Bruders in vollem Masse zu erreichen. Beide Zwillingsbrüder spielen heute mit gleicher Spielstärke in der ersten Tennismannschaft ihres Heimatortes. 
Seit dem im Alter von 10 Jahren erlittenen Unfall fühlte sich II stets gegenüber seinem Bruder zurückgesetzt. Die Rivalität zwischen den Brüdern, die erst im letzten Jahr (mit 19 Jahren) ein erträgliches Verhältnis zueinander gefunden haben, führte dazu, dass sie in verschiedenen Schulen (I Realgymnasium, II Waldorfschule) aufgenommen werden mussten. Beide sind mit anderen gute Doppelpartner, niemals aber zusammen.
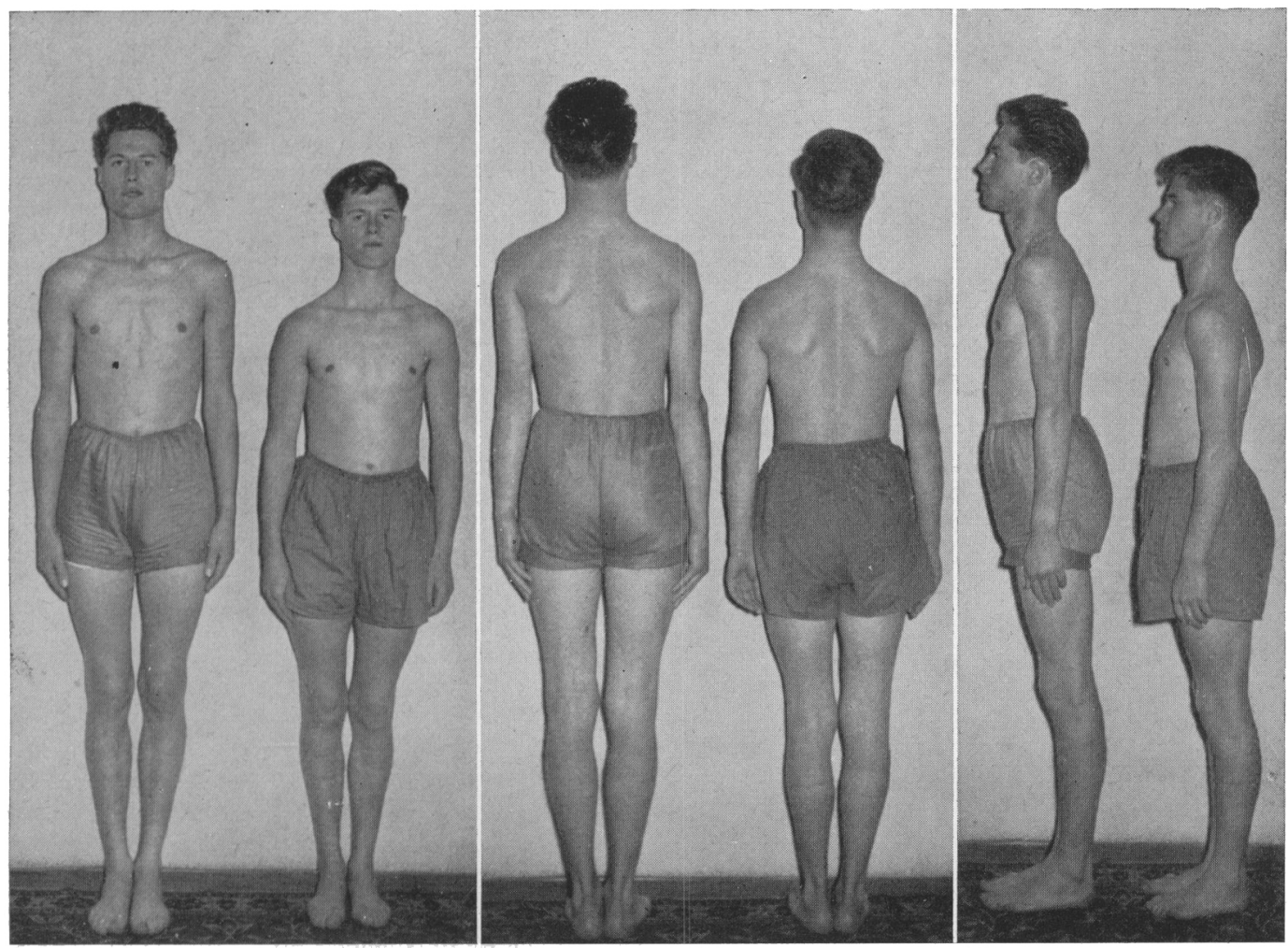

Abb. 4 - Durch Kryptorchismus (?) des Kleineren ausgelöste körperliche und sportliche Leistungsverschiedenheit (KD) bei erbgleichen Zwillingen (EZ 13)

Die grosse Aehnlichkeit dieser Zwillingsbrüder kommt auch — trotz des Armwechsels des verletzten - beim Tennis in der Schlägerhaltung, in der Reaktion während des Spiels an der Grundlinie oder am Netz und im Spielaufbau zum Ausdruck. Die anthropologischen Masse dieser Zwillingsbrüder stimmen fast völlig überein.

EZ 131936 geboren und bisher stets in gleicher Umwelt. Beide leben mit ihrer Mutter, einer Kriegerwitwe, in besonders herzlichem Verhältnis zusammen; sie haben die gleichen Interessen und gehen in die gleiche Oberschulklasse.

Bei der körperlichen Untersuchung konnte ich bei den in Abb. 4 wiedergegebenen

19 - A. Ge. Me. Ge (IV). 
Brüdern im Alter von 18 Jahren bei II einen hypoplastischen linken Hoden feststellen, während der gleiche Hoden bei $\mathrm{I}$ in normaler Grösse entwicklet ist. Nach Angabe ist II mehrere Jahre lang wegen Kryptorchismus hormonal behandelt worden. Als Kinder sollen beide wegen weicher Leisten Bruchbänder getragen haben.

Aus den Lichtbildern geht die auch zahlenmässig bei der Festlegung der üblichen anthropologischen Masse gefundene Verschiedenheit zwischen den beiden Brüdern hervor, die sich in folgenden Werten ausdrückt (in $\mathrm{cm}$ ):

Tabelle II

\begin{tabular}{l|r|r}
\hline & I & II \\
\hline & 184,7 & 177,3 \\
Körpergrösse & 151,2 & 144,1 \\
Höhe des oberen Sternalrandes & 106,1 & 101,6 \\
Höhe des oberen Darmbeinstachels & 96,2 & 90,3 \\
Höhe des oberen Symphysenrandes & 34,9 & 34,5 \\
Kleinste Schulterbreite & 32,8 & 32,5 \\
Beckenbreite & 77,5 & 73,4 \\
Armlänge (rechts) & 98,5 & 91,5 \\
Beinlänge (rechts) & 18,3 & 17,4 \\
Grösste Kopflänge & 15,0 & 14,6 \\
Grösste Kopfbreite & 11,9 & 11,9 \\
Morphol. Gesichtshöhe & 18,5 & 18,3 \\
Physiognom. Gesichtshöhe & 13,4 & 13,1 \\
Jochbogenbreite & 11,4 & 11,2 \\
Kieferwinkelbreite & 6,0 & 5,9 \\
Ohrlänge (rechts) & 4,4 & 4,3 \\
Ohrbreite (rechts) & &
\end{tabular}

Abgesehen von der körperlichen Unterschiedlichkeit zeigen diese beiden EZ-Brüder im Gegensatz zu allen übrigen EZ-Paaren auch in der sportlichen Leistungsfähigkeit deutlichere Verschiedenheiten. Zwar treiben beide in gleicher Weise Leichtathletik, Handball, Fussball, Tennis und Tischtennis. Doch bestehen Unterschiede in der Bevorzugung besonderer Lieblingssportarten wie auch in der Leistungshöhe.

In der Leichtathletik bevorzugt I Wurfübungen und Laufstrecken bis $800 \mathrm{~m}$, II er zielte seine bisher besten Leistungen in Mittelstrecken- $u$. Langstreckenläufen, besonders bei Waldläufen. Während I ein besserer Tennisspieler ist als II, hat es II im Tischtennis zu grösserer Fertigkeit gebracht.

Als Erklärung für die morphologische und sportlich aktive Verschiedenheit dieser Zwillingsbrüder, die sich besonders gut vertragen und auch gleichartige Schulleistungen aufweisen, scheint mir das bei II durch einen Kryptorchismus gestörte hormonale Gleichgewicht eine ausreichende Begründung zu geben. Künftige Untersucher möchte ich deshalb auf die Beachtung entsprechender Befunde hinweisen. 
Weiblich :

EZ 141936 geboren. Bis zur Schulentlassung im Elternhaus, seitdem als Büroangestellte in verschiedenen Betrieben tätig. Beide Schwestern werden oft verwechselt (s. Abb. 5). Schon während der Schulzeit waren beide hervorragende Leichtathletinnen, die bei den Schulsportfesten ihrer Heimatstadt Kassel wiederholt die beiden ersten Plätze erringen konnten. Dabei bestanden in der Punktwertung meist nur geringe Unterschiede nach der einen oder anderen Seite, mehrfach wurde völlige Punktgleichheit erreicht.

Seit 3 Jahren sind beide aktive Schwimmerinnen. Sie gehören zu den besten Schwimmerinnen Hessens. I ist besser im Kraulund Rückenschwimmen, II erzielt bessere Zeiten im Brustschwimmen.

Der Vater dieser EZ-Schwestern war als Leichtathlet und Schwimmer jahrelang sportlich aktiv.

EZ 151938 geboren. Im gleichen Schwimmverein wie EZ 14. Ebenfalls als Wettkampfschwimmerinnen im Kraul- und besonders im Rückenschwimmen eingesetzt. Beide sind Untersekundanerinnen mit ähnlichen Schulleistungen und gleichen Lieblingsfächern. Wie EZ 14 zeigen auch EZ 15 eine solch grosse Aehnlichkeit, dass sie - besonders auch bei Wettkämpfen - oft miteinander verwechselt werden. Als Nebensport treiben beide Geräte- und Bodenturnen. Eine ältere Schwester ist ebenfalls Wettkampfschwimmerin. Der Vater war aktiver Ruderer und Leichtathlet.

EZ 161937 geboren. I im elterlichen

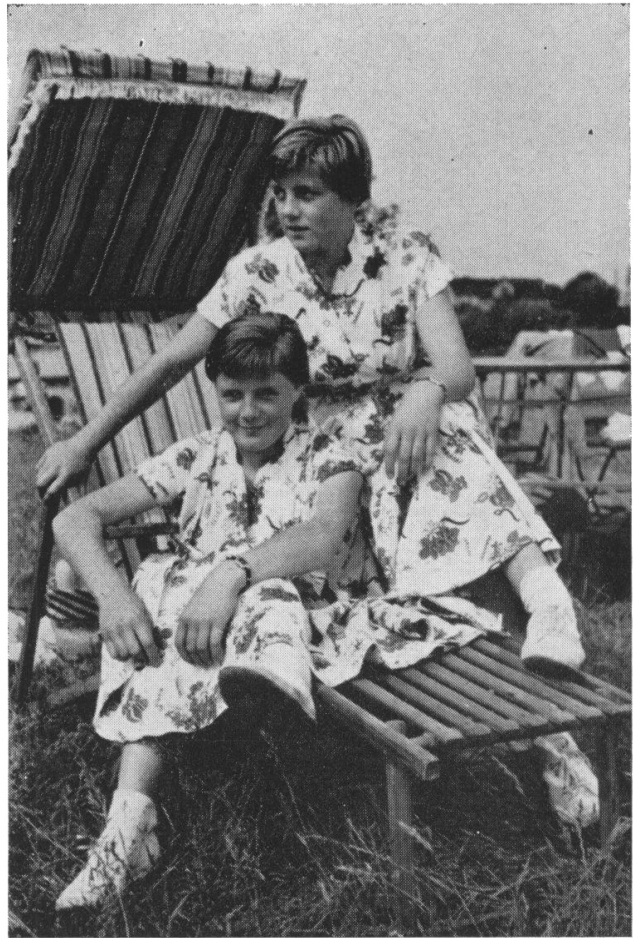

Abb. 5 - Erbgleiche Zwillingsschwestern mit gleichen Wettkampfleistungen im Schwimmen (EZ 14) Haushalt, II als Kindergärtnerin tätig. Neben gleichartiger musikalischer Begabung und gleichen Interessen sind beide überdurchschnittlich gut veranlagte Leichtathletinnen und vor allem Schwimmerinnen mit Bevorzugung des Brustschwimmens. Der Vater dieser EZ-Schwestern ist Lehrer mit besonderem Interesse für Musik und Sport. Er war in seiner ostpreussischen Heimat im Geräteturnen und Schwimmen auch in Wettkämpfen eingesetzt.

EZ 171927 geboren. Stammen aus einer ausgesprochenen Sportlerfamilie, mit EZ 3 verwandt. Der einzige Bruder ist aktiver Handballer und Turner, ebenso war der einzige 
Bruder der Mutter, dessen sämtliche 3 Kinder Leistunggportler sind, als Geräteturner and Schwerathlet besonders erfolgreich.

Die in Abb. 6 wiedergegebenen EZ-Schwestern, die zur Zeit als Torhüterin und Verteidigerin in der gleichen Handballmannschaft spielen, zählen zu den besten Gerätetur-

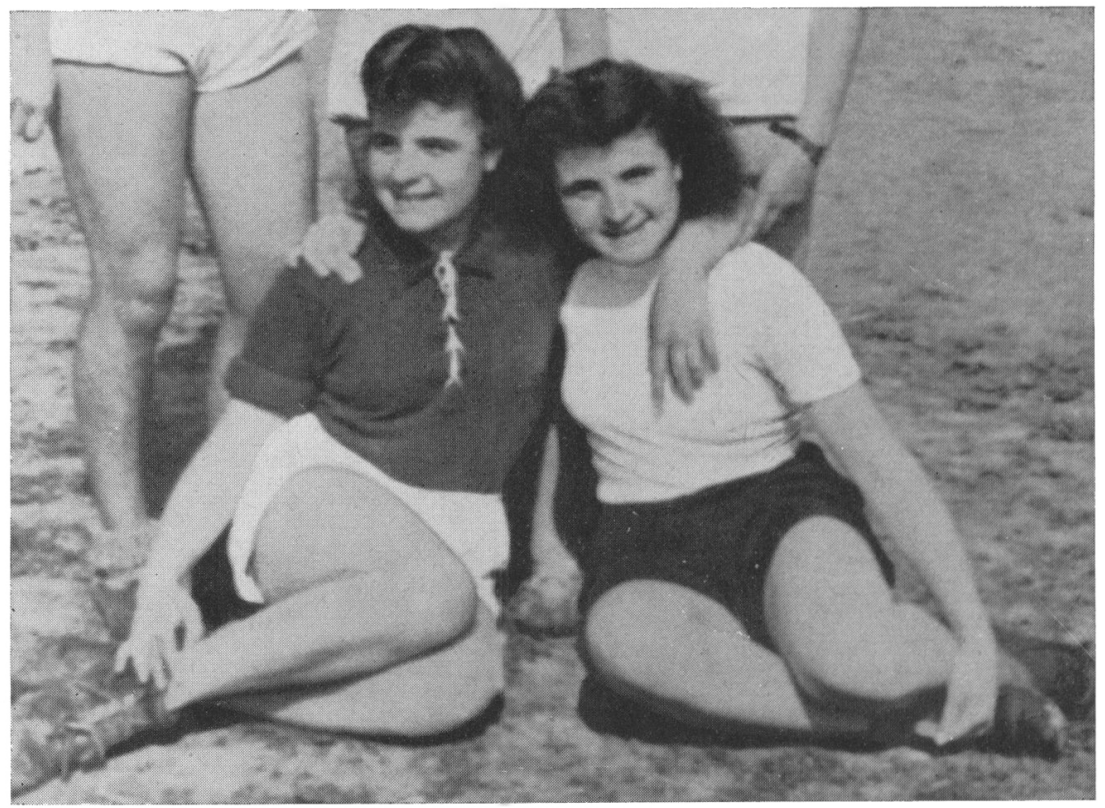

Abb. 6 - In der gleichen Handballmannschaft spielende erbgleiche Zwillingsschwestern, in deren Familie zahlreiche Sportler, darunter Zwillingsvettern (EZ 3) hervorgetreten sind (EZ 17)

nerinnen und Leichtathletinnen ihrer Heimatstadt. Auch in Volkstänzen sind die nicht nur im Habitus, sondern auch in Bewegung und Mimik sehr ähnlichen Schwestern hervorgetreten.

EZ 181936 geboren. Wie EZ 17 sind diese EZ-Schwestern überdurchschnittlich begabte Geräteturnerinnen und besonders erfolgreiche Handballspielerinnen. Schwimmen und Skilauf unterstreichen die sportliche Veranlagung der beiden Schwestern, deren Vater Vorstandsmitglied der berühmten Frankfurter « Eintracht » ist. Mit den Zwillingen spielt eine Base in der ersten Damenhandballmannschaft dieses Vereins, die wiederholt süddeutscher Meister war. In der Familie sind auch weitere Verwandte als Aktive (Fussballer) oder Funktionäre im gleichen Sportclub tätig.

EZ 191935 geboren. Seit Schulentlassung (mittlere Reife) als Büroangestellte (I Bank, II Wohnungsbaugesellschaft) tätig. Beide litten als Kind an Asthma bronchiale. Seit dem 14. Lebensjahr sind beide nach wiederholten Schwarzwaldkuren ohne Be- 
schwerden. Nachdem beide EZ-Schwestern bis auf Schwimmen und Radfahren keinen Sport getrieben hatten, begannen sie gleichzeitig mit Rollschuhlauf und konnten zwei Jahre später bei den Meisterschaften von Darmstadt die beiden ersten Plätze belegen. Auch bei den hessischen Meisterschaften erreichten sie mehrfach Vorderplätze. Die trotz unterschiedlicher Frisur ausserordentlich grosse Aehnlichkeit dieser Schwestern (Fingerhaltung!) geht aus Abb. 7 hervor.

Die Mutter war eine gute Geräteturnerin. Besondere sportliche Leistungen sind sonst in der Familie nicht erzielt — auch nicht versucht - worden.

EZ 201940 geboren. Trotz ihrer Jugend waren diese EZ-Schwestern bereits mehrfach in Jugendschwimmkämpfen mit nahezu gleichen Leistungen siegreich. In der väterlichen wie in der mütterlichen Familie sind $Z$ willingsgeburten vorgekommen. Wie die Zwillinge, die neben Schwimmen auch Geräteturnen betreiben, sind mehrere Verwandte Mitglieder von Turn- und Schwimmvereinen, allerdings ohne besondere Wettkampfneigung.

EZ 211938 geboren. Von den Eltern, die Pferdeliebhaber sind, wurden beide früh dem Reitsport zugeführt. Die Mutter war zudem eine gute Tennisspielerin. Beide EZSchwestern, die als Untersekundanerinen noch zur Schule gehen, haben bereits eine Reihe von Turniererfolgen aufzuweisen und gemeinsam auch mehrfach Reitschulen besucht. Die Aehnlichkeit der Schwestern, die ausser Schwimmen und etwas Tennis nicht sportlich tätig sind, geht auch aus dem Reitsitz und der Haltung bei Sprüngen hervor (Abb. 8).

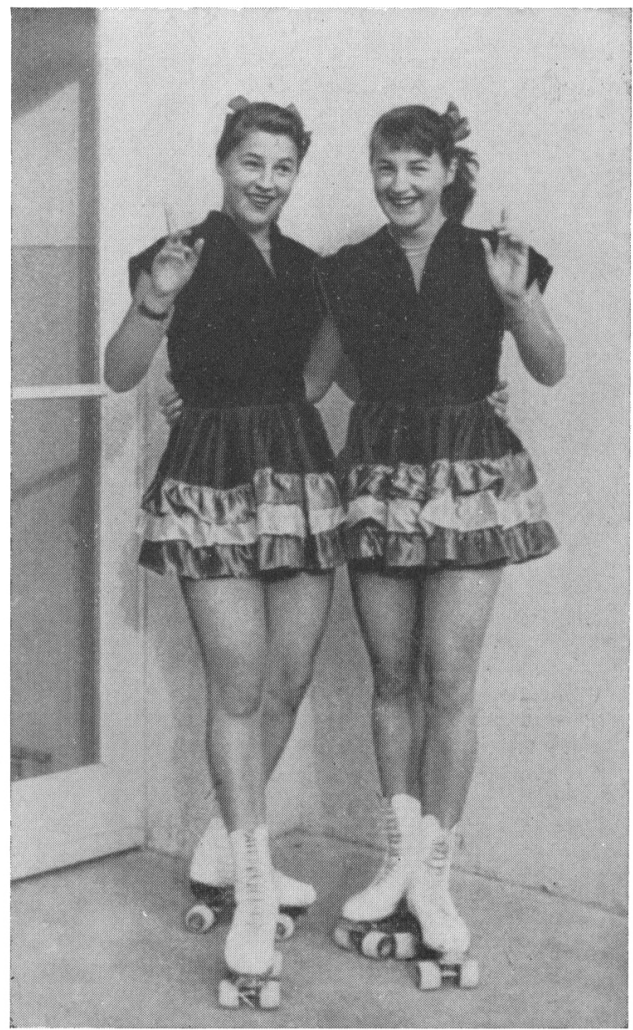

Ảbb. 7 - Erbgleiche Zwillingsschwestern mit gleichen Spitzenleistungen im Rollschuhlauf (EZ 19)

\section{Männlich :}

\section{b. Erbverschiedene Zwillinge (ZZ)}

ZZ 11933 geboren. Bei einem Grössenunterschied von $10 \mathrm{~cm}$ spielen beide Brüder seit 1947 stets in der gleichen Mannschaft Handball. I ist Medizinstudent, II landwirtschaftlicher Gehilfe. Der einzige Bruder der Zwillinge spielt zur Zeit als linker Läufer in der gleichen Amateurligamannschaft, in der I Mittelläufer und II Halbstürmer ist. 
Alle drei sind mittelgute Geräteturner. In der Familie haben die Mutter und der mütterliche Grossvater gut geturnt.

ZZ 21936 geboren. I ist Autoschlosser, II Bundesbahnangestellter. Wie der Vater, der früher Fussball-Ligaspieler war, sind beide sehr aktive Fussballer, zur Zeit als Halbstürmer in der gleichen Mannschaft. Als Nebensport treiben beide Rudern und Schwimmen, wollen aber in Zukunft nur noch Fussball spielen (Abb. 9).
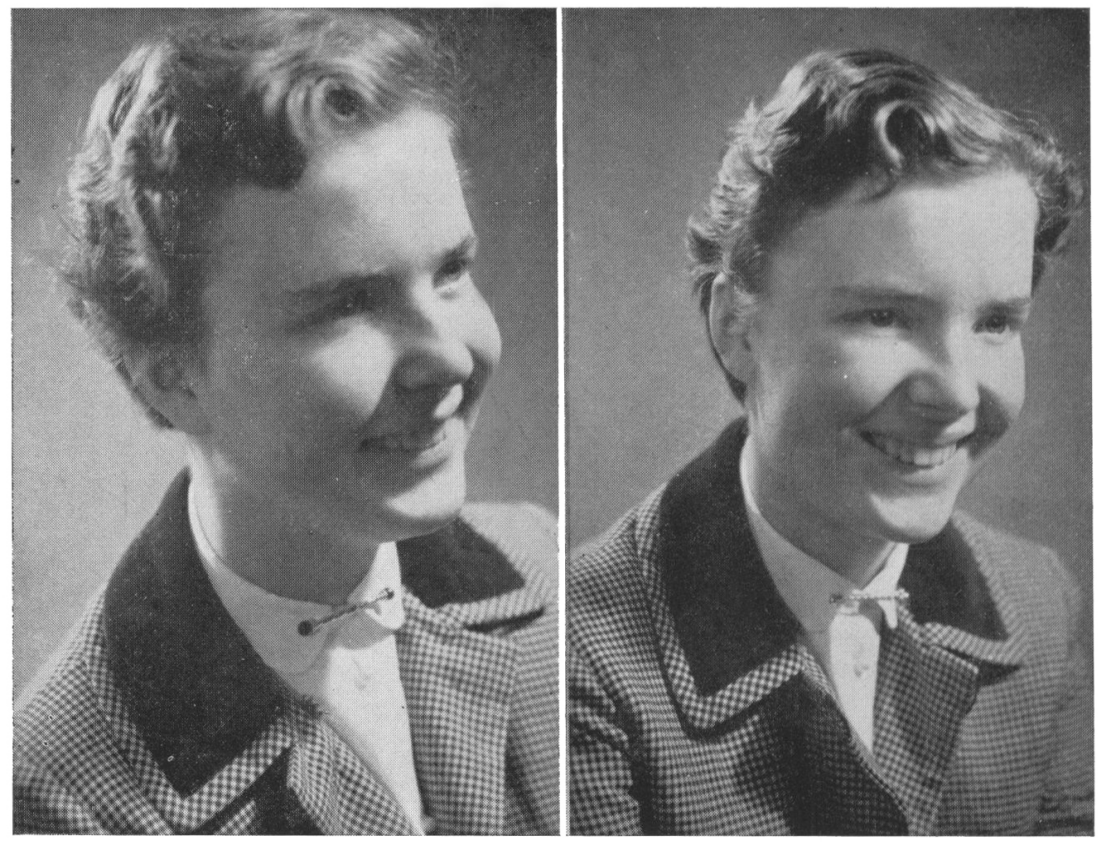

Abb. 8 - Reiterlich begabte erbgleiche Zwillingsschwestern mit gleichen Turniererfolgen (EZ 21)

ZZ 31935 geboren. Gehören zu den besten hessischen Schwimmern und Wasserballspielern und haben die Wasserballmannschaft des Kasseler SC 89 zur hessischen Meisterschaft geführt. Beide sind kaufmännische Angestellte. Ihre besten Schwimmzeiten waren:

$100 \mathrm{~m}$ Kraul I $1.06,5$, II $1.04,3$

$100 \mathrm{~m}$ Schmetterling I $1.20,0$, II $1.19,3$

$100 \mathrm{~m}$ Rücken I $1.18,5$, II $1.18,0$

Die einzige Schwester dieser ZZ-Brüder hat mehrere nordhessische Meisterschaften im Kraul- und Rückenschwimmen gewonnen. Der Vater, der u.a. Stadtmeister von Breslau im Tischténnis war, konnte 1935 an einem Tage 3 schlesische Schwimmeisterschaften (100 m Brust, $100 \mathrm{~m}$ beliebig, $50 \mathrm{~m}$ Rücken) erringen. 
ZZ 41936 geboren. In der Schule gute Turner. Seitdem aktive Handballer, zur Zeit in der gleichen hessischen Ligamannschaft als Läufer und Stürmer. In der Familie dieser ZZ-Brüder, die in der väterlichen Landwirtschaft tätig sind, waren keine weiteren Sportler.

ZZ 51939 geboren. I bei einem Gewicht von 77,5 kg 182 gross, II bei einem Gewicht von $73 \mathrm{~kg} 173 \mathrm{~cm}$ gross. 1953 errang I bei einem Leichtathletiksportfest (Kreismeister-
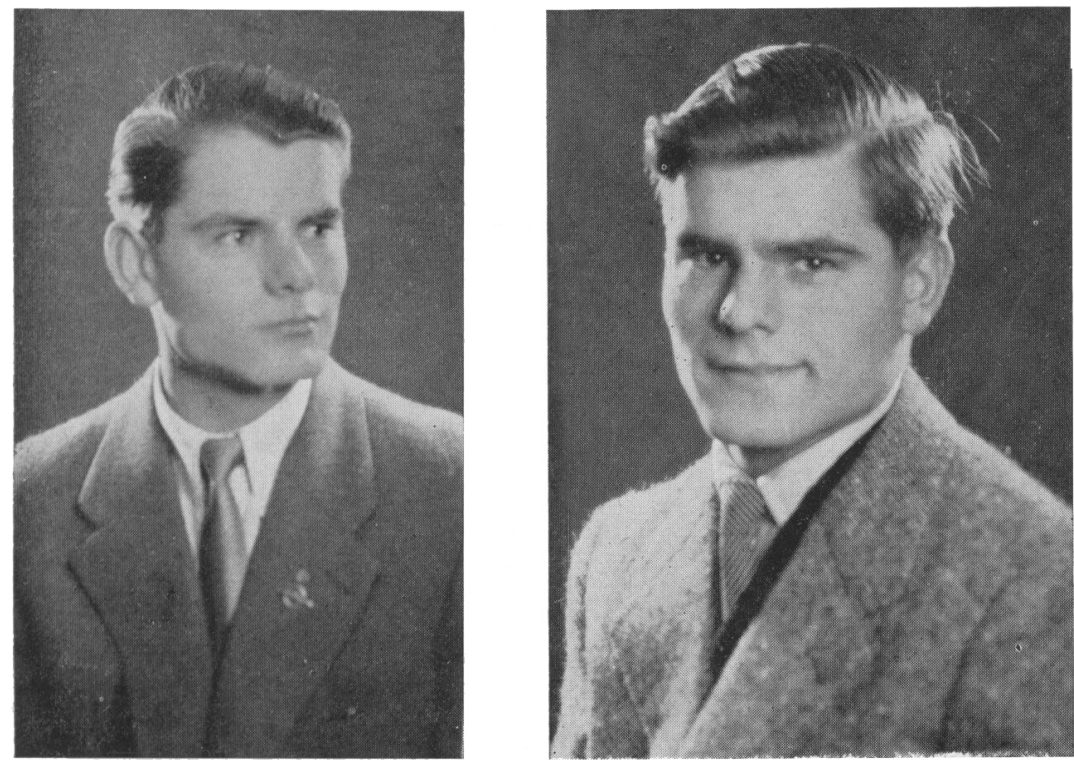

Abb. 9 - In der gleichen Fussballmannschaft aktive erbverschiedene Zwillingsbrüder ( $Z Z_{2}$ )

schaften) im Mehrkampf den 3., II den 7. Platz. Ein Jahr später errang I im gleichen Wettbewerb den 1., II dagegen nur den 12. Platz. 1954 errang I bereits in Wurfübungen und im Hochsprung Bezirksmeisterschaften, während II im Laufen und Weitsprung nur Mittelplätze erreichen konnte (Abb, 10).

ZZ 61932 geboren. Von diesen Brüdern hat, nachdem beide in der gleichen Jugendfussballmansschaft waren, der eine bis zum 19. Lebensjahr Fussball gespielt, der andere ist jetzt noch Mitglied eines Radfahrervereins und nimmt auch noch am Amateurrennen teil. In der Familie spielt ein jüngerer Bruder Fussball.

ZZ 71937 geboren. Von diesen sehr verschiedenen Zwillingsbrüdern geht I noch als Primaner in die Oberschule, während II mit der mittleren Reife auf eine Werkschule ging. Schon in der gemeinsamen Schulzeit war I, der einer der besten Läufer seiner Heimatstadt ist, ein weitaus besserer Sportler als II, der seit seinem 14. Lebensjahr in- 


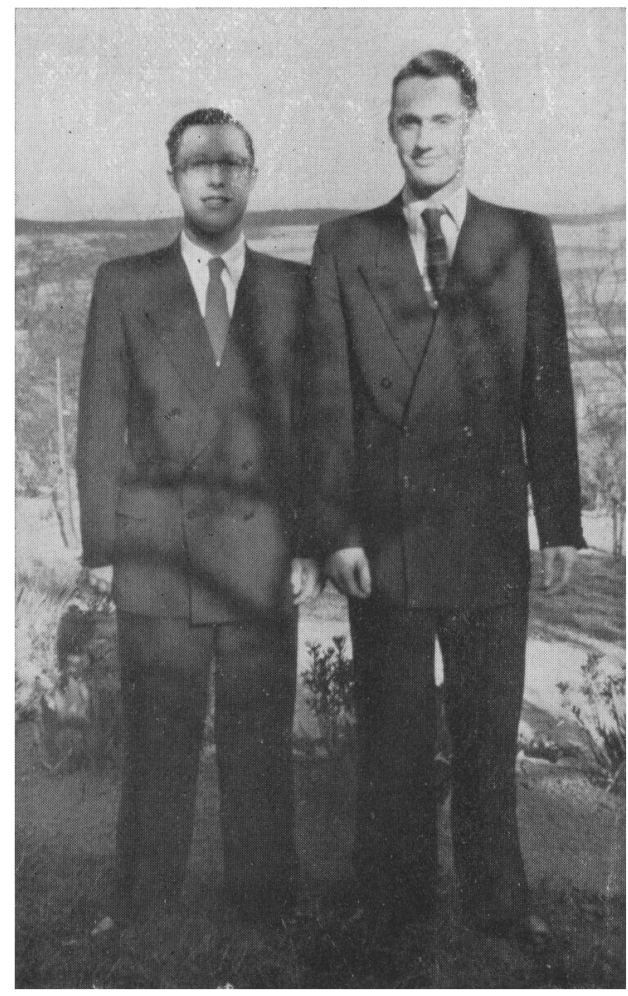

Abb. 10 - Erbverschiedene Zwillingsbrüder mit unterschiedlichen Leistungen (KD) in der Leichtathletik ( $\mathrm{ZZ}$ 5)

tensiv Musik studierte. I hat die Absicht, Sportlehrer $z u$ werden, II ist mehr an Musik und Kunstgewerbe interessiert. In der musikalisch begabten Familie sind Leistungssportler nicht hervorgetreten.

\section{Weiblich :}

ZZ \& 1935 geboren. Relativ ähnliche ZZ, die bereits in wiederholten Aehnlichkeitsuntersuchungen im früheren Frankfurter Institut meines Lehrers $v$. Verschuer als erbverschieden (zweieiig) diagnostiziert worden waren. Bei der letzten Untersuchung in der elterlichen Wohnung habe ich bei I $169 \mathrm{~cm}$ Körpergrösse und $62 \mathrm{~kg}$ Gewicht und bei II 168 cm Körpergrösse und 58,5 kg Gewicht feststellen können (Abb. 11).

Beide Schwestern, die aus einer ausgesprochenen Sportlerfamilie stammen, haben

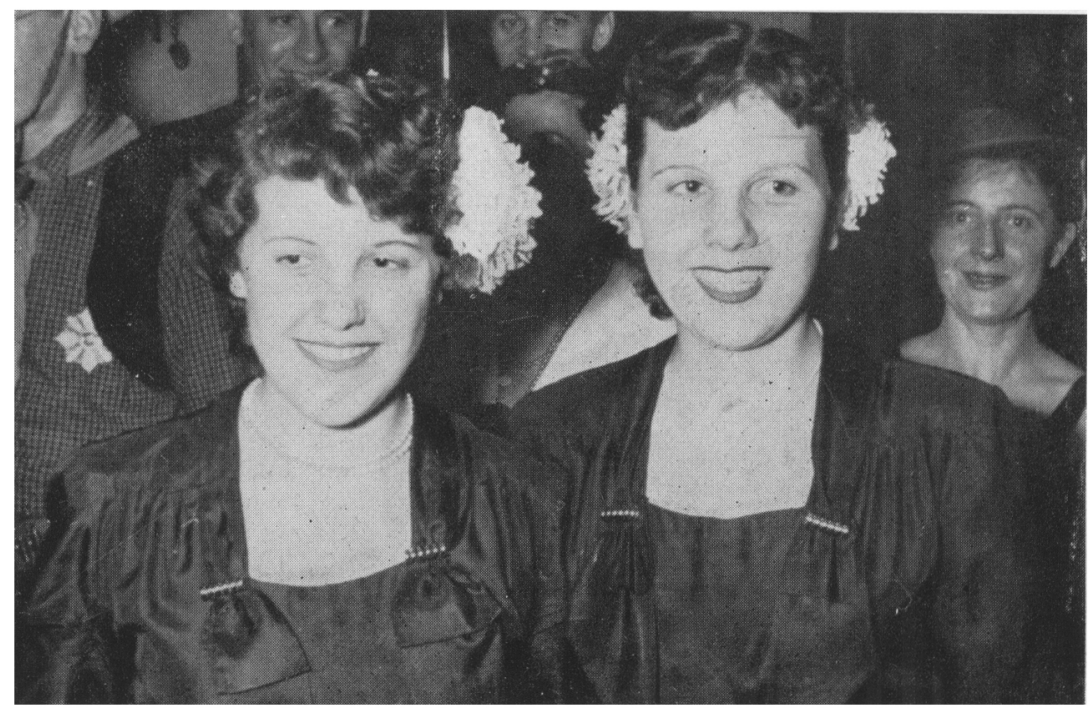

Abb. 11 - Erbverschiedene Zwillingsschwestern mit gleicher sportlicher Leistungshöhe aus einer Sportlerfamilie (ZZ 8) 
zahlreiche leichtathletische Erfolge, darunter 1951 bei den hessischen Jugend-Waldlaufmeisterschaften die beiden ersten Plätze (II wurde Meisterin) erreichen können. In der süddeutschen Handball-Meistermannschaft der Frankfurter Eintracht spielt I Mittelstürmer und II Mittelläufer.

Die Zwillingsschwestern haben 6 lebende Geschwister, die durchweg überdurchschnittliche sportliche Leistungen aufweisen, 2 Brüder sind Radfahrer; sie konnten 1954 bei den deutschen Jugendmeisterschaften den 2. und 6. Platz erreichen. 2 weitere Brüder sind aktive Handballer und Fussballer. Von den beiden Schwestern der Zwillinge spielt die eine Handball, die andere ist eine erfolgreiche Leichtathletin. Der Vater dieser Geschwister konnte als Soldat den damaligen deutschen $100 \mathrm{Me}$ ter-Meister schlagen und 1948 in russischer Gefangenschaft bei einem Leichtathletiksportfest 7 erste Preise gewinnen.

ZZ 91939 geboren, Arztkinder. Beide haben seit dem 10. Lebensjahr an zahlreichen Sportwettkämpfen, besonders leichtathletischen, teilgenommen. Dabei wurde I, die mit 14 Jahren $8 \mathrm{~cm}$ grösser als die bis dahin fast

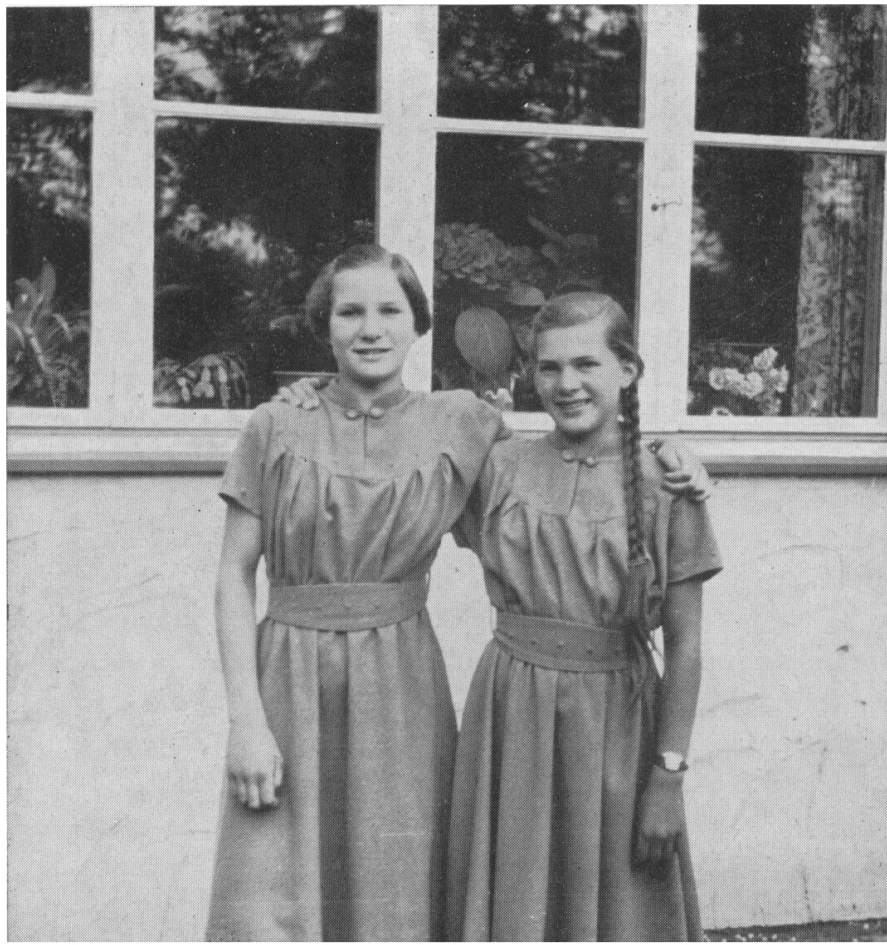

Abb. 12 - Trotz starker körperlicher Verschiedenheit in ähnlicher Weise sportlich begabte erbverschiedene Zwillingsschwestern (ZZ 9)
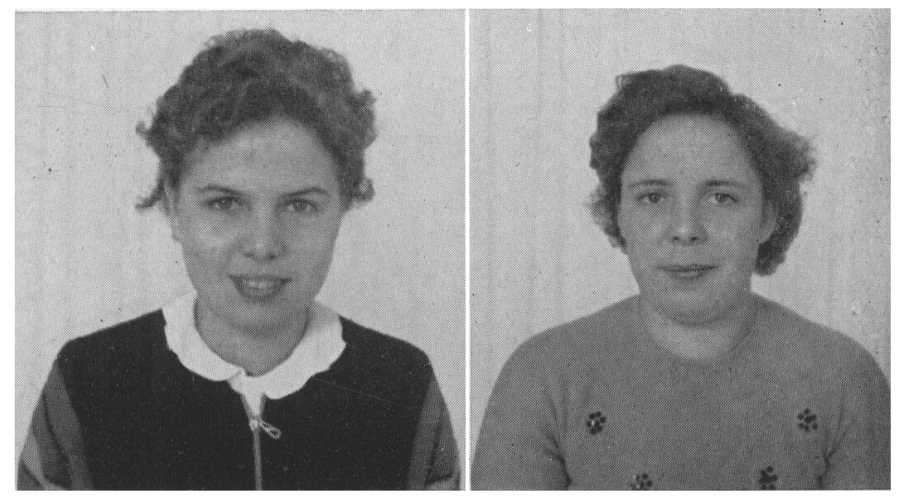

Abb. 13 - Zweieiig 2 Zwillingsschwestern mit unterschiedlichen Interessen und Leistungen ( $Z Z$ 11, II hat ein Kind.) 
gleichgrosse Schwester wuchs, meist Siegerin ihrer Altersklasse und II Zweite (Abb. 12). Neben dem Sport mit besonderen Leistungen auch im Ski- und Schlittschuhlauf, im Schwimmen und im letzten Jahr vor allem auch im Tennis (I wurde Jugendmeisterin des Kreises Waldeck vor II) sind beide Schwestern auch musikalisch begabt (I spielt Geige, II Klavier). In der Freizeit interessiert sich I für Tiere, II für Technik.

In der Familie haben mehrere Angehörige - ohne besonderen Ehrgeiz — Tennis gespielt.

ZZ 101937 geboren, Oberschülerinnen der gleichen Klasse, die bisher stets gemeinsam im Elternhaus waren und auch gemeinsam Sport, hauptsächlich Schwimmen und früher Handball, betrieben haben. In Wettkämpfen sind beide bisher wenig hervorgetreten. Der Vater war früher erfolgreicher Wettschwimmer.

ZZ 111932 geboren. Schon in der Schule war II $(161,5 \mathrm{~cm}$ gross, $74 \mathrm{~kg}$ Gewicht, verheiratet, seit 1953 eine Tochter) stets eine bessere Turnerin als I (158 cm gross, 57,5 kg Gewicht, ledig, kinderlos). Nach der Schulzeit betrieb I Bodenturnen und Schwimmen. In einem Wettbewerb in Kassel errang sie im Streckentauchen den ersten und im Brustschwimmen den zweiten Platz. Sie hat nie gern an Geräten geturnt, II hat dagegen seit ihrem 15. Lebensjahr in einer Leistungsriege geturnt (Lieblingsgerät Barren) und nie an einem Schwimmwettbewerb teilgenommen; sie hat nie Strecken tauchen können (Abb. 13).

In der Familie hat der Vater jahrelang in einer 1. Fussballmannschaft gespielt. Der einzige Bruder der Schwestern ist Ruderer.

ZZ 121915 geboren. Beide sind bereits mit 5 Jahren Freischwimmer und Mitglieder eines Turnvereins gewesen. Mit 9 Jahren waren beide bereits gute Weitspringerinnen. Nachdem II damals bereits $3.90 \mathrm{~m}$ weit gesprungen war, musste sie wegen Kreislaufstörungen jeden Sport aufgeben. Mit 12 Jahren lernten beide Schwestern Skilaufen. Während I sich von dieser Zeit an hauptsächlich dem Skilauf, Bergsteigen und später dem Segelfliegen widmete, kam II nach vorübergehenden Tenniserfolgen mit 18 Jahren zur Leichtathletik und mit 19 Jahren zum Wettkampfsport. Nach Erringung der deutschen Meisterschaft im $80 \mathrm{~m}$-Hürdenlauf kam sie 1936 bei den Olympischen Spielen in den Endlauf dieser Disziplin. 1938 war sie deutsche Studentenmeisterin und 1942 nach 4 . jähriger Pause noch Zweite bei den deutschen Meisterschaften. Sie ist Sport- und Gymnastiklehrerin und jetzt mit einem sportärztlich tätigen Orthopäden verheiratet (Abb. 14). Alle Familienangehörigen sind sportlich interessiert, jedoch nicht aktiv hervorgetreten.

ZZ 131926 geboren. Beide hatten in der Schule stets sehr gute Turnnoten. Als sie mit 14 Jahren gemeinsam begannen, Leichtathletik zu betreiben, erzielte II (Ilse) zunächst bessere Leistungen als I, zog sich aber, da sie Sport nicht mehr interessierte, bald ganz von jeder sportlichen Betätigung zurück. I (Margot) wurde jedoch nach systematischem Training in den Jahren 1947 bis 1952 eine der besten deutschen Leichtathletinnen. Sie gewann 1950 die deutsche Meisterschaft im 100 m Lauf und war nach zahlreichen Hallensiegen 1952 gemeinsam mit ihrem Ehemann, der eine Medaille im 800 m-Lauf gewinnen 

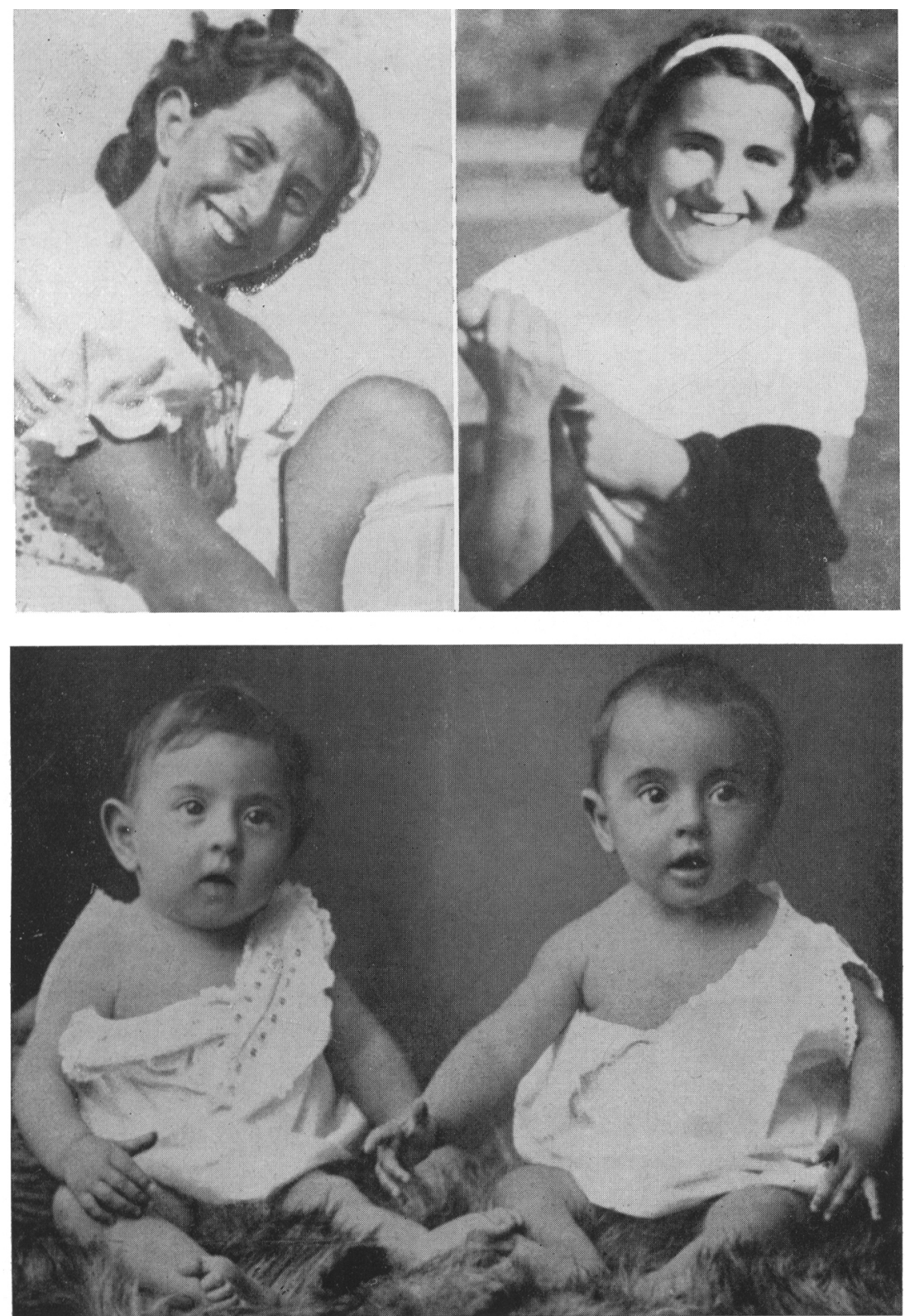

$c$

Abb. 14a, b, c-Verschieden sportlich interessierte (KD), erbverschiedene Zwillingsschwestern (ZZ 14: I (a) Bergsteigerin und Segelfliegerin, II (b) Deutsche Meisterin und Olympiateilnehmerin im $80 \mathrm{~m}$-Hürdenlauf. (c) Kinderbild der Schwestern 
konnte und nach mehreren deutschen Meisterschaften auch amerikanischer Hallenmeister geworden war, Olympiateilnehmerin in Helsinki. Angaben über sportliche Leistungen bei Blutsverwandten konnte ich nicht erhalten.

Wenn auch die Gesamtzahl der von mir bisher untersuchten 34 gleichgeschlechtigen Zwillingspaare nicht als gross und repräsentativ angesprochen werden kann und meine Erhebungen zur Gewinnung einer nach Möglichkeit auslesefreien Zwillingsserie durch

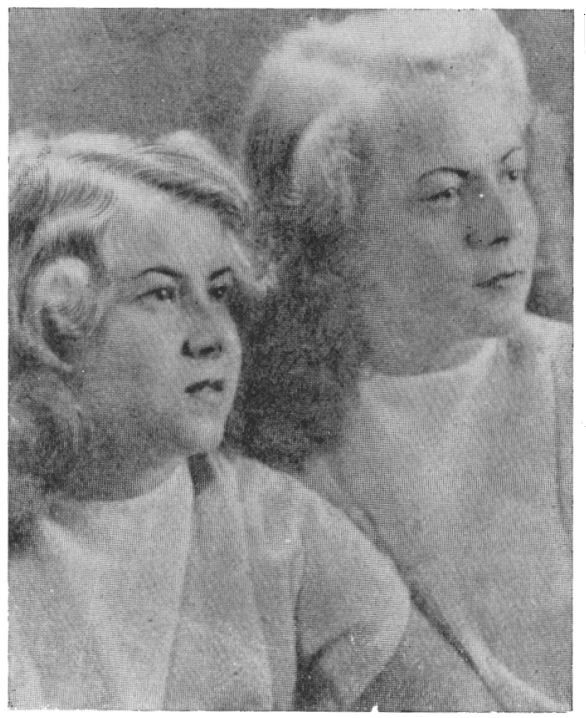

Abb. 15 - Erbverschiedene Zwillingsschwestern, von denen I (links) deutsche Meisterin und Olympiateilnehmerin im 100 m-Lauf war, während II (rechts) sportlich völlig inaktiv ist ( $Z Z 13$ ) Ueberwiegen der EZ doch eine gewisse Auslese darstellen, so darf doch nach den mitgeteilten Befunden gefolgert werden:

1. Völlige Diskordanz in der Einstellung dem Sport gegenüber wie in der sportlichen Aktivität konnte bei insgesamt 21 EZ-Paaren nicht beobachtet werden.

Während alle übrigen EZ-Paare sowohl in der sportlichen Aktivität als auch in der be. vorzugten Sportart und der erzielten sportlichen Leistung sich völlig konkordant verhielten, fanden sich lediglich bei dem EZ-Paar 13 nennenswerte Verschiedenheiten in den erzielten sportlichen Leistungen und den bevorzugten sportlichen Disziplinen bei völlig gleichartigem sportlichem Interesse. Die Ursache des diskordanten Verhaltens in Bezug auf die Leistung in einzelnen Sportarten ist wohl in der vor allem in der unterschiedlichen Körpergrösse zum Ausdruck kommenden konstitutionellen Verschiedenheit dieser EZ-Brüder zu sehen. Die Unterschiede in der äusseren Körperform dieser Zwillingsbrüder lassen sich aber mit einer Hodenatrophie des Kleineren der Brüder nach früherer Kryptorchismusbehandlung zurückführen. Wir dürfen damit eine wahrscheinlich nicht in der Erbmasse begründete Erklärung für die partielle Diskordanz der EZ 13 in ihrer sportlichen Leistungsfähigkeit in Betracht ziehen.

Die überraschend hohe Konkordanz der übrigen EZ-Paare könnte man - abgesehen von der Erbgleichheit - auch auf die meist gleiche Umwelt und den Ansporn zurückführen, der in den meisten Familien durch das Beispiel der Eltern und anderen Verwandten gegeben ist.

Als Beweis dafür, dass aber doch die Erbmasse entscheidend für die angetroffenen Konkordanzen bei den sporttreibenden EZ-Paaren in Erscheinung tritt, möchte ich das EZ-Paar 12 ansehen. Von den mit EZ-Paar 13 fast gleichaltrigen Brüdern des EZ-Paares 12 erlitt der eine im Alter von 10 Jahren durch Verlust der rechten Hand (Handgranaten- 
explosion) ein schweres körperliches und psychisches Trauma, das auch zu einer mehrere Jahre anhaltenden Sprachstörung geführt hat. Erst nach Umschulung auf die unverletzt gebliebene Hand konnte der geschädigte Bruder in einen echten sportlichen Wettbewerb mit dem unverletzten Bruder treten und nach nunmehr 10 Jahren die volle Leistungshöhe des Bruders erreichen. Die Erbmasse, die sich auch in gleichem Leistungsniveau beim Fussball und beim Reiten zeigt, war in diesem Falle so stark, dass nach Ueberwindung des körperlichen und psychischen Schadens, die Jahre in Anspruch nahmen, ein bedeutender Vorsprung des nicht verletzten Bruders aufgeholt werden konnte.

Welch andere Deutung kann in diesem Falle für die heute sehr eindrucksvolle gleiche Spielstärke und Spielauffassung beider Brüder bei einer der schwierigsten Sportarten, beim Tennis, gegeben werden?

Nachdem die - wahrscheinlich in Minderwertigkeitskomplexen des Verletzten zu suchenden - seelischen Spannungen zwischen den mir gut bekannten EZ-Brüdern 12 in den letzten beiden Jahren geringer geworden sind, darf angenommen werden, dass in absehbarer Zeit auch eine weitere seelische Angleichung der körperlichen folgen wird, wie sie bereits in der Sportausübung recht deutlich geworden ist.

Zusammenfassend sprechen die erhobenen Befunde bei EZ dafür, dass der Erbveranlagung für die Art der sportlichen Betätigung wie auch für die erzielbare Leistungshöhe eine grössere Bedeutung zukommen muss, als dies bisher angenommen werden konnte oder bekannt war.

2. Im Gegensatz zu der hohen Konkordanz in der Sportausübung wie den erzielten sportlichen Leistungen bei den EZ, fanden sich bei 6 vom 13 ZZ-Paaren mehr oder weniger grosse Diskordanzen. Dabei ist hervorzuheben, dass neben 4 ZZ-Paaren mit ähnlichem sportlichem Interesse, jedoch unterschiedlicher Ausübung und Leistungshöhe in den bevorzugten Sportarten, auch 2 ZZ-Paare ( 1 männliches und 1 weibliches) mit so grosser Diskordanz angetroffen wurden, dass eine Einordnung in die Spalte D (Diskordanz in der Einstellung zum Sport wie in der sportlichen Betätigung und Leistungsfähigkeit) berechtig war.

Von den ZZ-Paaren mit starker Diskordanz zeigte nur einer der Brüder bleibendes Interesse am Sport wie am Leistungssport, während der andere sich stark musikalisch betätigt; bei dem Schwesternpaar hat es die ursprünglich offenbar weniger begabte Schwester bis zu einer deutschen Meisterschaft gebracht, während die andere jegliche sportliche Neigung früh aufgegeben hat. Es ist möglich, dass bei dem Schwesternpaar mit starker Diskordanz die heute noch sportlich aktive Schwester durch die Bekanntschaft und spätere Ehe mit einem Meistersportler besonders gefördert worden ist. Die Trennung der sportlichen Interessen dieser beiden Schwestern erfolgte jedoch bereits während des Pubertätsalters.

Zusammenfassend zeigen die ZZ-Zwillingspaare in ihrer Einstellung dem Sport gegenüber wie in der sportlichen Aktivität und im erreichten Leistungsniveau gegenüber den EZ so deutliche Verschiedenheiten, dass auch die Konkordanz-Diskordanzunterschiede bei EZ einerseits und $\mathrm{ZZ}$ andererseits die Abhängigkeit der Ausübung der einen oder anderen Sportart ganz allgemein wie etwa möglicher besonderer sportlicher Leistungen von der Erbmasse unterstreichen. 
Die von vielen Seiten bestrittene Bedeutung des Genotypus für das Zustandekommen einer herausragenden sportlichen Leistung kommt in den Unterschieden bei den von uns untersuchten sporttreibenden Zwillingen mit mehr oder weniger grossen sportlichen Erfolgen eindeutig zum Ausdruck. Durch die hiermit vorgelegten ersten Ergebnisse einer versucht auslesefreien Zwillingsuntersuchung dürfen auch die bisherigen Befunde in Familien mit mehreren gleichartig hervorgetretenen Sportlern damit erklärt werden, dass der Erbmasse in solchen Familien neben der günstigen Umwelt ebenfalls eine entscheidende Bedeutung zuerkannt werden muss.

Ueber die Erhebungen in "Sportfamilien " soll in einer späteren Mitteilung berichtet werden.

\section{Zusammenfassung}

Es wird über die Ergebnisse einer ersten Zwillingsuntersuchung bei 34 gleichgeschlechtigen, sporttreibenden Zwillingspaaren berichtet, von denen 21 eineiig (erbgleich, EZ) und 13 zweieiig (erbverschieden, ZZ) waren. Während sich von den 13 EZ-Paaren 12 in ihrer sportlichen Betätigung wie in den erzielten sportlichen Leistungen völlig konkordant $(\mathrm{K})$ verhielten und nur 1 Paar geringe Unterschiede in der bevorzugten Sportart wie in der Leistungshöhe zeigte (KD), waren von den 13 ZZ-Paaren nur 7 völlig konkordant (K). Von den 6 ZZ-Paaren mit Diskordanzen zeigten 4 (2 männlich, 2 weiblich) deutlichere Leistungsunterschiede oder Verschiedenheiten in der bevorzugten Sportart (KD); von den restlichen beiden Paaren ( 1 männlich, 1 weiblich) ist jeweils nur ein Paarling Leistungssportler (darunter sogar eine deutsche Meisterin!), der andere ist sportlich völlig inaktiv.

Die deutlichen Konkordanz-Diskordanzunterschiede bei den untersuchten EZ und ZZ beweisen, dass der Erbmasse für die Einstellung zum Sport wie für die sportliche Leistungsfähigkeit eine nicht wegzuleugnende Bedeutung zukommt.

Auf die Notwendigkeit ausgedehnterer Zwillingsuntersuchungen zur Bestätigung oder Widerlegung der vorgelegten Befunde wird hingewiesen. 


\section{RIASSUNTO}

Vengono riferiti $\mathrm{i}$ risultati di una prima inchiesta che riguarda 34 coppie gemellari unisesso praticanti lo sport, di cui 2 I $\mathrm{MZ}$ e $13 \mathrm{DZ}$. Delle 13 coppie $\mathrm{MZ}$, I2 si comportano in modo totalmente concordante $(K)$ nelle loro manifestazioni sportive come pure nella forma sportiva conseguita e soltanto una coppia dimostra una piccola differenza nello sport preferito e nella capacità ad esercitarlo.

Soltanto 7 delle 13 coppie DZ sono completamente concordanti. Delle 6 coppie DZ (4 maschili e 2 femminili) con discordanze, 4 dimostrano evidenti differenze nello sport preferito o nel rendimento sportivo (KD).

Nelle 2 coppie restanti (I maschile, I femminile) soltanto un membro pratica attivamente uno sport (una gemella è perfino campionessa tedesca) mentre l'altro membro è inattivo. Le differenze relative alla concordanza e alla discordanza fra $\mathrm{MZ}$ e $\mathrm{DZ}$ esaminati dimostrano che la massa ereditaria ha un valore considerevole per l'interesse sportivo come pure per la capacità sportiva.

Questo fatto indica la necessità di un'inchiesta ben più vasta su coppie di gemelli non selezionate.

\section{SUMMARY}

Report of the results of an examination on 34 pairs of same sex twins with special activity in sport. 2I pairs were identical (EZ), 13 non identical (ZZ). While from the 13 identical pairs (EZ) I2 were quite of the same sporting activity as even the same performance (complete concordance, $\mathrm{K}$ ) and only one pair showed some difference in the favoured kind of sports as in the capacity (KD). From the 13 non identical pairs (ZZ) only 7 were of complete concordance (K). 4 ( 2 male, 2 female) of the 6 non identical pairs (ZZ) with discordances showed clearer differences in their sporting performance or in the favoured branch of sport (KD). From the last two pairs (I male, I female) only one of the twins was respectively famous in sport (even one German champion!), the other thereover of a complete sporting inactivity.

The distinct differences in concordance and discordance at the examined $E Z$ and $Z Z$ prove, that the genotypus is of undeniable importance for sporting interest and activity as for sporting performance.

In order to confirm or to refute the produced results it seems to be necessary to examine more extended series of non selected twins.
RÉSUMÉ

Rapport des résultats d'une première enquête de 34 jumeaux de même sexe faisant du sport, dont 2 I étaient identiques (EZ) et 13 pas identiques (ZZ). De I3 EZ-paires I2 se comportent complètement concordants (K) dans leurs manifestations sportives comme dans la puissance sportive atteinte et seulement I paire montre une petite différence dans le sport préféré comme dans la capacité.

Il y a seulement 7 des 13 paires $Z Z$, qui sont complètement concordants $(\mathrm{K})$. Des 6 paires $\mathrm{ZZ} \mathrm{(4} \mathrm{mascul.,} 2$ fémin.) avec discordances 4, montrent clairement différences dans le sport préféré ou dans la puissance sportive (KD). Des 2 paires qui restent (I mascul., I fémin.) seulement un des jumeaux est actif en sport (parmi desquels - même une championne allemande!), l'autre est sportif complètement inactif. Les différences distinctes de concordance et discordance sur les $\mathrm{EZ}$ et $\mathrm{ZZ}$ examinés demontrent que la masse héréditaire a une valeur considerable pour l'intérêt du sport comme pour la capacité sportive. Cela indique la nécessité d'une enquête sur les jumeaux bien plus grande pour accueil ou la réfutation des constatations montrées. 